\title{
Nova Tocha de Plasma Híbrida para o Processamento de Materiais
}

\author{
(New Hybrid Plasma Torch for Materials Processing)
}

\author{
Richard Thomas Lermen", Ivan Guerra Machado ${ }^{2}$ \\ ${ }^{1}$ Faculdade Horizontina (FAHOR), Departamento de Engenharia Mecânica, Horizontina, RS, Brasil, richard@fahor.com.br \\ ${ }^{2}$ Universidade Federal do Rio Grande do Sul (UFRGS), PPGE3M /LS\&TC, Porto Alegre, RS,Brasil, welder@ufrgs.br
}

\begin{abstract}
Resumo
O principal objetivo deste artigo foi apresentar um novo dispositivo para o processamento de materiais. Ele consiste em uma tocha de plasma híbrida, a qual é caracterizada pela formação simultânea de dois arcos plasma em apenas um dispositivo, gerando jato (de plasma) com elevada densidade de energia. A tocha foi submetida aos seguintes testes experimentais: de funcionamento para verificar possíveis problemas de projeto e seus limites de operação; de caracterização, consistindo em determinar o comprimento do jato de plasma; de sua viabilidade para processamento de materiais (soldagem e corte). Com base nestes testes iniciais, alguns problemas de isolamento elétrico e térmico foram encontrados e resolvidos. Quanto aos resultados dos testes de caracterização, os parâmetros de funcionamento da tocha de plasma hibrida apresentaram influência significativa sobre o comprimento do jato de plasma. Os resultados obtidos nos testes de processamento de materiais foram satisfatórios, ou seja, é possível realizar soldagem e corte com esta tocha de plasma hibrida.
\end{abstract}

Palavras-chave: Tocha de Plasma Hibrida; Jato de Plasma; Soldagem; Corte Térmico.

\begin{abstract}
The main aim of this paper was to present a new device for materials processing. It consist of a hybrid plasma torch which is characterized by the simultaneous formation of two plasma arcs in one device only, generating a (plasma) jet with high energy density. The torch was submitted to the following trials: of operation to identify possible design problems and its operational torch limits; of characterization, consisting in plasma jet length determination; and of viability for materials processing (welding and cutting). Based on these initial trials, some electrical and thermal insulation problems were found and solved. Concerning the results of the characterization trials, the hybrid plasma torch parameters had a significant influence over the plasma jet length. The results obtained in the materials processing trials were satisfactory, i.e., it is possible to carry out welding and cutting with this hybrid plasma torch
\end{abstract}

Key-words: Hybrid Plasma Torch; Plasma Jet; Welding; Thermal Cutting.

\section{Introdução}

Os plasmas térmicos gerados por meio de tochas específicas ("tochas de plasma") tiveram suas primeiras aplicações tecnológicas na década de 1960. Nos últimos anos, essa tecnologia tornou-se uma das mais promissoras, pois apresenta várias aplicações e pode ser uma das menos poluentes [1, 2]. Entre as tochas específicas encontram-se as tochas híbridas geradoras de plasma, as quais são caracterizadas por utilizar simultaneamente dois ou mais processos em apenas um dispositivo [3] e, por exemplo, tocha para soldagem Plasma/ MIG [4], tocha de plasma com duplo anodo [5, 6], entre outras

(Recebido em 16/02/2012; Texto final em 30/07/2012).
[7]. Estas tochas geralmente são desenvolvidas visando a obtenção de vantagens competitivas em relação aos processos de fabricação convencionais.

As tochas de plasma geram jatos de gás ionizado que produzem, em geral, fluxos de gás com elevada temperatura e com densidade de potência de, aproximadamente, $10^{8} \mathrm{~W} / \mathrm{m}^{2}$. Com este jato de plasma é possível elevar à temperatura de fusão ou vaporização quase todos os materiais, sendo a utilização de jatos de plasma para soldagem e corte relativamente bem descrita na literatura [por exemplo, 8-10].

Recentemente [11] um Propulsor Magnetoplasmadinâmico ou MPDT (sigla em inglês para "Magnetoplasmadynamic Thruster"), como é mais conhecido, foi desenvolvido, produzido e aperfeiçoado com o intuito de realizar soldagem e corte de materiais metálicos, no Laboratório de Soldagem \& Técnicas Conexas (LS\&TC) da Universidade Federal do Rio Grande do Sul (UFRGS). Porém, este dispositivo ainda não apresentou soldagens e cortes com qualidades comparáveis aos processos convencionais (TIG, MIG/MAG etc.). 
Não existindo informações suficientes disponíveis a respeito da potencialidade do MPDT, quando aliado aos outros processos de soldagem e tecnologias de fontes de potência, um novo dispositivo híbrido gerador de plasma foi desenvolvido, o qual envolve o processo MPDT e o processo plasma nãotransferido [12]. Observa-se que este dispositivo tem Pedido de Patente efetuado pela UFRGS junto ao Instituto Nacional de Propriedade Industrial, cujo número definitivo é PI 1104137-4, com o título "Tocha Híbrida Geradora de Plasma para Aplicação em Processos de Fabricação, e, Processo de Produção de Tocha", sendo os inventores Richard Thomas Lermen e Ivan Guerra Machado.

Para esta nova tocha de plasma híbrida, os conhecimentos científicos relacionados com as características do jato de plasma (curvas características de tensão e corrente, comprimento do jato de plasma, força propulsora resultante, distribuição de temperatura etc.) e a aplicação em processos de fabricação (soldagem, corte, endurecimento superficial etc.) ainda não foram relatados, nem de forma superficial, sendo os resultados aqui encontrados fundamentais para o desenvolvimento científico-tecnológico deste assunto.

\section{Materiais e Métodos}

Os materiais e métodos são apresentados em três etapas, isto é, a primeira descreve o projeto e funcionamento da tocha de plasma híbrida, a segunda apresenta os métodos e materiais para a sua caracterização e, por fim, é descrito a aplicação da tocha em soldagem e corte. O objetivo desta última etapa não foi otimizar procedimentos com o novo dispositivo mas, sim, verificar a sua adequação para soldagem e corte de materiais.

\subsection{Projeto e Funcionamento da Tocha de Plasma Híbrida}

A tocha de plasma híbrida caracteriza-se pela formação simultânea de dois arcos plasmas em apenas um dispositivo, o qual gera um jato de plasma com elevada densidade de energia, sendo resultado da combinação sinérgica de dois processos geradores de plasma, ou seja, Plasma Não-Transferido e Propulsor Magnetoplasmadinâmico (MPDT).

Esta tocha possui uma geometria coaxial constituída por dois catodos, primário e secundário, e dois anodos cilíndricos em forma de câmara, os quais são isolados eletricamente. Inicialmente, um gás é injetado através de orifícios gerando um fluxo na câmara, onde o gás é aquecido e ionizado através da passagem pela descarga elétrica entre os eletrodos (catodo primário e anodo primário), gerada por uma fonte primária de energia com dispositivo de alta frequência. Através deste plasma (gás ionizado) surge uma densidade de corrente radial, a qual atravessa o gás em direção ao catodo primário. Esta corrente no catodo primário gera um campo magnético induzido circunferencial, o qual interage com a densidade de corrente aparecendo uma força eletromagnética, geralmente denominada "força de Lorentz", a qual acelera as partículas formando uma câmara de plasma.

Sendo o plasma um condutor elétrico, outra descarga elétrica é gerada por uma fonte secundária de energia entre o catodo secundário e anodo secundário na câmara. Uma segunda densidade de corrente radial surge atravessando o plasma em direção ao catodo secundário, onde esta corrente no catodo secundário também gera um campo magnético induzido circunferencial, que, por sua vez, interage com a densidade de corrente secundária aparecendo outra força eletromagnética , a qual ioniza e acelera ainda mais as partículas do jato de plasma provenientes da primeira ionização e também é responsável pela expulsão do jato de plasma para fora da câmara.

Portanto, neste sistema o gás é acelerado duas vezes, isto é, primeiro pela força eletromagnética entre os eletrodos primários e, sequencialmente, pela força eletromagnética resultante entre os eletrodos secundários.

Um esquema da tocha de plasma híbrida com a representação das linhas de densidade de corrente elétrica é mostrado na Figura 1 .

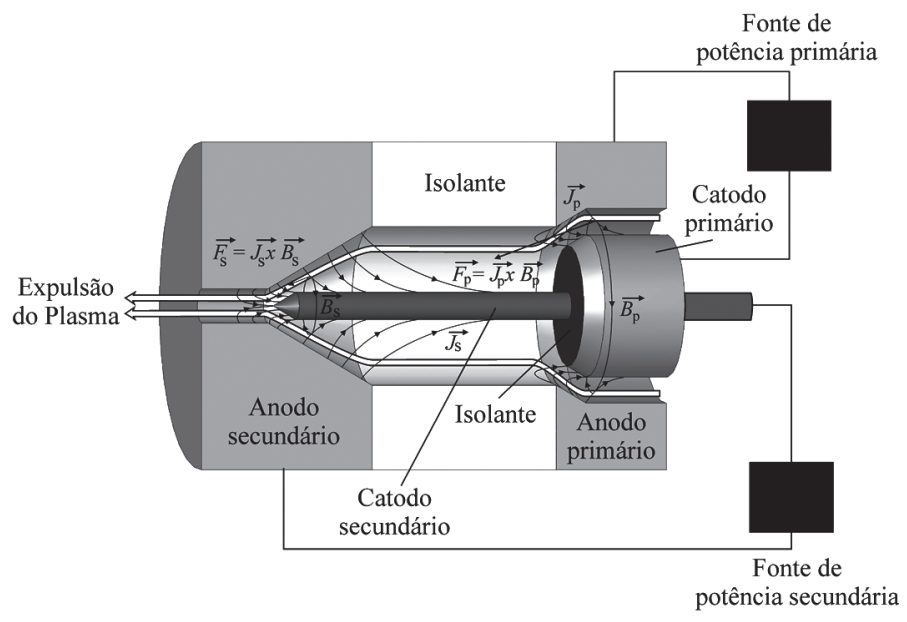

Figura 1. Desenho esquemático da tocha de plasma híbrida.

Por sua vez, a Figura 2 mostra um desenho esquemático da configuração inicial da tocha de plasma híbrida. Este dispositivo é composto principalmente por dois catodos, primário e secundário. $\mathrm{O}$ catodo secundário é um eletrodo de tungstênio puro (classe AWS EW) com $8 \mathrm{~mm}$ de diâmetro, $150 \mathrm{~mm}$ de comprimento e extremidade cônica de $60^{\circ}$, enquanto o catodo primário é um eletrodo confeccionado em cobre com forma de anel. Os anodos primário e secundário são ambos confeccionados em cobre, formando uma câmara.

Os eletrodos são fixados em câmaras refrigeradas com água e isolados eletricamente entre si através de isolantes usinados em forma de flanges. As dimensões foram determinadas de acordo com a literatura sobre transferência térmica, eletromagnetismo e, principalmente, dinâmica dos fluidos em tocha de plasma $[2$, 13-16]. As câmaras de refrigeração são encaixadas em flanges de cobre, os quais são fixados com parafusos e porcas. Buchas de teflon são utilizadas para evitar descargas elétricas entre os parafusos e os flanges. O gás de trabalho é adicionado à tocha através de duas entradas (primária e secundária). A entrada de gás secundária foi criada, principalmente, para auxiliar na refrigeração do catodo secundário. 


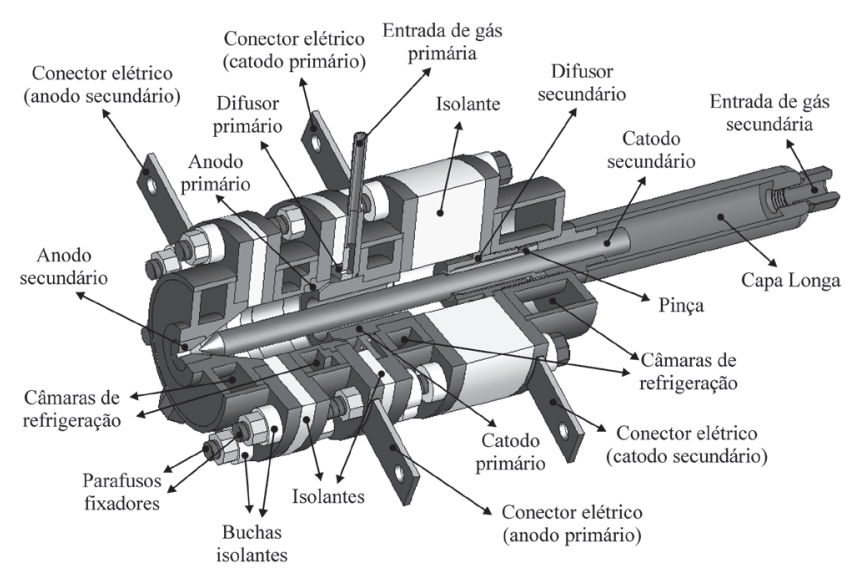

Figura 2. Desenho esquemático inicial da tocha de plasma híbrida.

Devido aos problemas de isolamento elétrico e deficiência na refrigeração apresentados no projeto inicial da tocha, algumas modificações foram realizadas, as quais podem ser vistas na Figura 3, que apresenta a tocha de plasma híbrida modificada.

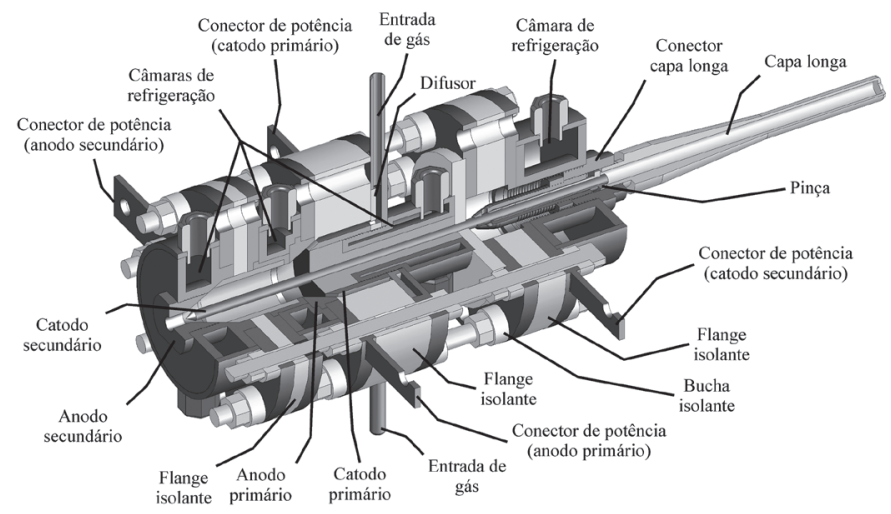

Figura 3. Tocha de plasma híbrida modificada.

Nesta nova configuração, o catodo primário foi modificado para um eletrodo em forma de câmara com íntimo contato com a água, aumentando a sua refrigeração. A câmara de refrigeração do anodo secundário e o anodo secundário foram alterados melhorando o resfriamento do eletrodo. Também, para melhorar a refrigeração dos eletrodos, duas entradas de água foram adicionadas, sendo uma para a primeira e segunda câmara de refrigeração e a outra para a terceira e quarta câmara de refrigeração, diferentemente do projeto inicial, no qual havia apenas uma entrada de água para as quatro câmaras de refrigeração. $\mathrm{O}$ catodo secundário de tungstênio puro com $8 \mathrm{~mm}$ de diâmetro foi substituído por um eletrodo classe AWS EWTh2 com diâmetro de $3,2 \mathrm{~mm}$. As deficiências no isolamento elétrico foram resolvidas através das seguintes modificações: todos os parafusos foram isolados dos flanges de cobre com buchas de teflon; um bocal cerâmico (mesmo tipo usualmente utilizado no processo de soldagem TIG) foi anexado entre os eletrodos (anodo primário e anodo secundário) para evitar a instabilidade do arco elétrico primário; a entrada de gás primária foi excluída devido à necessidade de isolar o catodo primário do catodo secundário, sendo este isolamento feito com uma bucha de material cerâmico.

Duas fontes de potências de característica corrente constante ("tombante") foram utilizadas para o acionamento da tocha de plasma híbrida. Estas fontes são distintas quanto aos intervalos da intensidade de corrente elétrica e aplicação, isto é, a fonte de potência primária possui um intervalo de corrente elétrica de 0 a $70 \mathrm{~A}$, um gerador de alta frequência e é apropriada para o processo de corte térmico a plasma, enquanto que a fonte de potência secundária tem intervalo de corrente elétrica de 0 a 400 A e é utilizada para o processo de soldagem TIG.

O sistema de injeção do gás na tocha de plasma é composto por um cilindro de argônio com válvula de diafragma e fluxômetro, os quais controlam e medem o fluxo de entrada de gás dentro da tocha de plasma. $\mathrm{O}$ gás argônio foi admitido diretamente na câmara de descarga da tocha pelas entradas de gás indicadas na Figura 3. Com este sistema, vazões de até 25 1/min de argônio foram possíveis. Por sua vez, a refrigeração dos eletrodos foi realizada através de câmaras refrigerantes, as quais eram alimentadas por água pela rede externa, formando um sistema aberto de refrigeração.

\subsection{Caracterização da Tocha de Plasma Híbrida}

A caracterização do dispositivo consistiu em determinar os efeitos dos parâmetros de funcionamento (vazão do gás e intensidades de corrente - primária e secundária) sobre o comprimento do jato de plasma gerado.

Os experimentos para a caracterização do dispositivo foram conduzidos de acordo com um projeto de experimentos, o qual foi elaborado através do método estatístico fatorial completo. Este projeto possui três variáveis (intensidade de corrente elétrica primária $-I_{P}$, intensidade de corrente elétrica secundária $-I_{S}$ e vazão do gás $-V_{g}$ ) com quatro níveis (valores). Os níveis utilizados foram: intensidade da corrente elétrica primária de $30 \mathrm{~A}, 40 \mathrm{~A}, 50 \mathrm{~A}$ e $60 \mathrm{~A}$; intensidade da corrente elétrica secundária de 100 A, 150 A, 200 A e 250 A; vazão do gás de $7 \mathrm{l} / \mathrm{min}, 14 \mathrm{l} / \mathrm{min}, 20 \mathrm{l} / \mathrm{min}$ e $25 \mathrm{l} / \mathrm{min}$. Os níveis para cada variável foram escolhidos através de experimentos prévios, os quais foram realizados, principalmente, para determinar as limitações do funcionamento da tocha de plasma híbrida. As vazões do gás foram determinados de acordo com as limitações do fluxômetro utilizado. A ordem de execução dos experimentos foi aleatoriamente determinada para evitar erros sistemáticos e três repetições dos experimentos foram feitas para reduzir o erro. As medidas de intensidade de corrente elétrica e aquisição de imagens foram realizadas simultaneamente, totalizando 192 experimentos.

Os comprimentos dos jatos de plasma foram determinados através da análise de imagens, as quais foram adquiridas com uma máquina fotográfica digital. As imagens foram processadas através do software "imageJ" e os efeitos dos parâmetros sobre o comprimento do jato de plasma foram determinados através de análise de variância (ANOVA). 


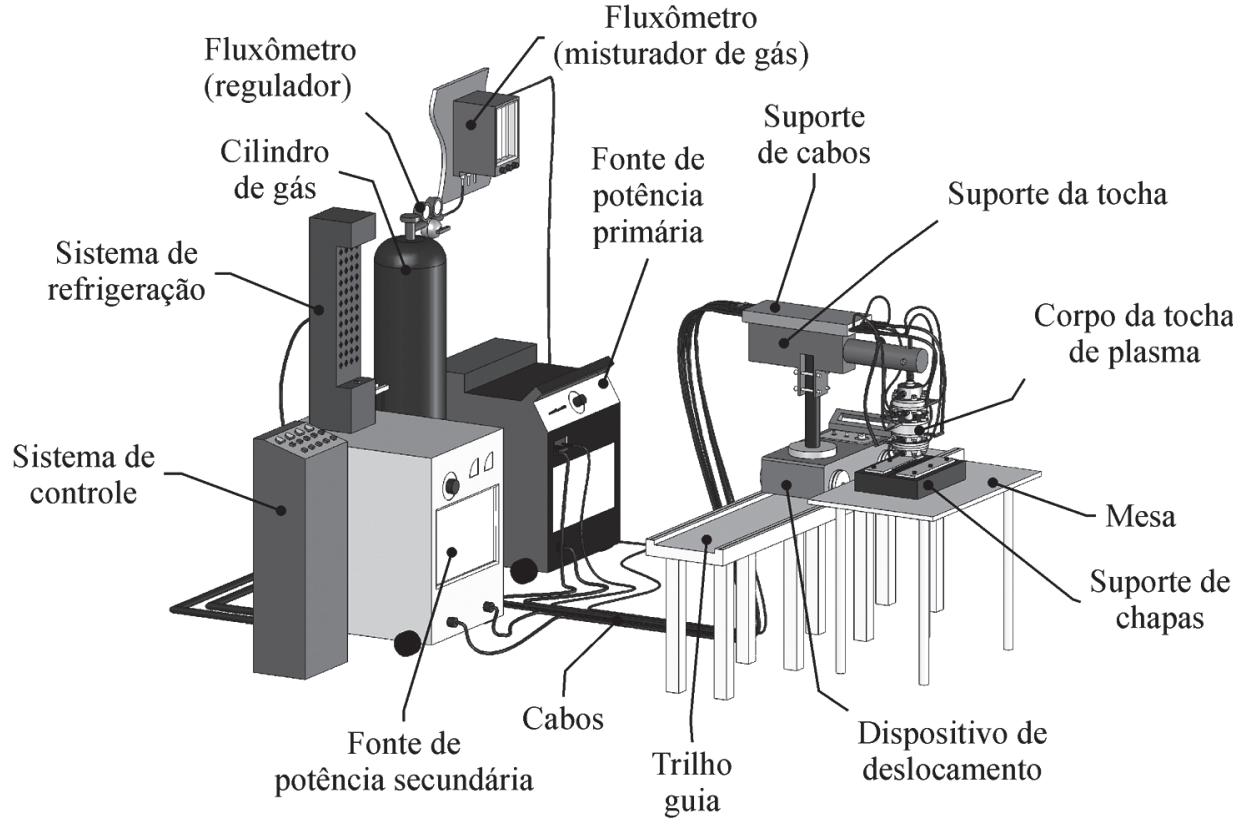

(a)

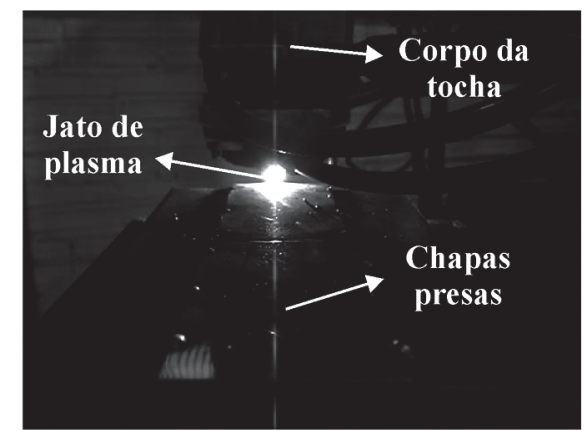

(b)

Figura 4. (a) Bancada utilizada nos testes de soldagem e corte; (b) Tocha funcionando em um dos testes de soldagem.

\subsection{Soldagem e Corte com a Tocha de Plasma Híbrida}

A aplicação da tocha de plasma híbrida no processamento de materiais consiste em testar a possibilidade de realização de soldagem e corte. Para a realização dos testes de soldagem e corte foi utilizado um sistema de bancada, o qual é constituído pelos seguintes equipamentos: fontes de potência; sistema de deslocamento; suportes para fixar a tocha e os cabos; cilindro de gás, reguladores de gás; sistema de controle, e outros. A Figura 4(a) ilustra o esquema da bancada utilizada nos testes e a Figura 4(b) mostra a tocha de plasma híbrida em operação em um dos testes de soldagem.

Os testes de soldagem foram realizados utilizando chapas de diferentes espessuras $(0,7 \mathrm{~mm}$ a $6,35 \mathrm{~mm})$ e materiais (SAE 1020 e AISI 304). As chapas foram fixadas e centralizadas de acordo com o jato de plasma exaustado para fora da câmara. Os parâmetros de soldagem utilizados foram os seguintes: distância entre o bocal e a peça $5 \mathrm{~mm}$; gás argônio injetado na câmara da tocha com vazões de $7 \mathrm{l} / \mathrm{min}$ a $25 \mathrm{l} / \mathrm{min}$; intervalos de intensidade da corrente elétrica das fontes de potência primária e secundária de $30 \mathrm{~A}$ a $60 \mathrm{~A}$ e $100 \mathrm{~A}$ a $250 \mathrm{~A}$, respectivamente; velocidade de soldagem de $1,7 \mathrm{~mm} / \mathrm{s}$ a $8,3 \mathrm{~mm} / \mathrm{s}$; ângulo na ponta do catodo secundário $60^{\circ}$; distâncias entre os eletrodos primários $3 \mathrm{~mm}$ e distância entre os eletrodos secundários $2 \mathrm{~mm}$.

Os testes de corte com a tocha de plasma híbrida foram realizados usando chapas de diferentes espessuras $(0,5$ à $4 \mathrm{~mm})$ e materiais (alumínio e vários tipos de aços). Gás argônio foi injetado na câmara do dispositivo com vazão acima de $20 \mathrm{l} /$ min. A distância entre o bocal de saída do jato de plasma e a peça foi de $5 \mathrm{~mm}$, tendo sido utilizadas diferentes velocidade de deslocamento da tocha e intensidades da corrente elétrica (fonte primária e secundária).

\section{Resultados e Discussões}

\subsection{Testes Iniciais com a Tocha de Plasma Híbrida}

Os testes iniciais consistiram em analisar a funcionalidade da tocha de plasma híbrida, isto é, analisar o funcionamento da tocha quanto à formação dos arcos elétricos (primário e secundário) e jatos de plasmas, vazamentos de gás, isolamentos elétricos e térmicos, entre outros.

Nos primeiros testes com a tocha de plasma foram constatados alguns problemas que ocasionaram o funcionamento da tocha em condições precárias. Primeiramente, foi verificado que ocorriam vazamentos de gás entre algumas conexões da tocha gerando instabilidade nos arcos elétricos. Este problema foi resolvido através da adição de silicone para alta temperatura entre as partes em que ocorriam vazamentos de gás.

Em relação à formação dos arcos elétricos (primário e secundário) individualmente, ambos apresentaram jato de plasma aparentemente estável. A Figura 5 apresenta o jato de plasma obtido com a tocha de plasma híbrida parcialmente montada, isto é, o jato de plasma gerado pelo arco elétrico primário (arco não-transferido). Neste caso, foi observado que o ponto quente ("hot spot") do arco elétrico formado entre o anodo primário e o catodo primário fazia movimentos aleatórios em relação aos eletrodos. Também, os jatos de plasma gerados pelo arco secundário apresentaram características semelhantes aos jatos obtidos por estudos anteriores com propulsores magnetoplasmadinâmicos [11]. 


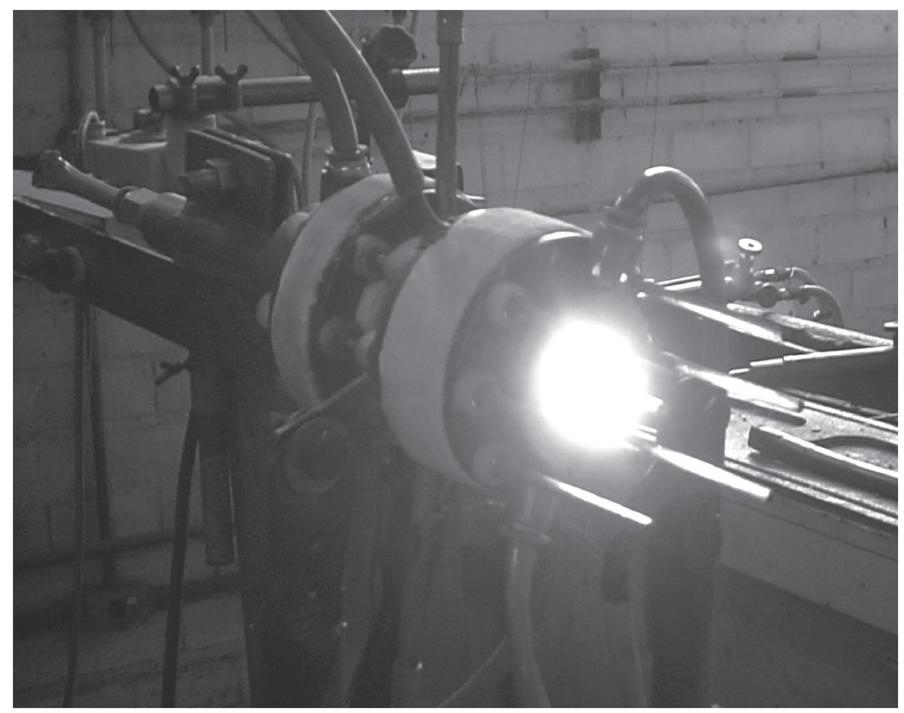

Figura 5. Tocha de plasma híbrida funcionando com apenas o arco elétrico primário.

Outro problema constatado foi insuficiente refrigeração do catodo primário e do anodo secundário, nos quais ocorria excessivo desgaste por erosão, com a Figura 6 apresentando alguns eletrodos utilizados e danificados devido a este fenômeno. Para resolver este problema, optou-se pela construção de novos eletrodos. No caso do anodo secundário, inicialmente o eletrodo de cobre (Figura 6(d)) foi substituído por um eletrodo de tungstênio (Figura 6(e)) com as mesmas dimensões; porém, persistiram os efeitos da deficiente refrigeração. Então, o diâmetro interno da câmara de refrigeração foi aumentado, consequentemente, outro anodo secundário (Figura 6(f)) foi desenvolvido, onde a parede de cobre entre a água e o eletrodo foi diminuída, aumentando assim a troca de calor entre eles. A excessiva erosão do catodo primário foi eliminada através da construção de uma nova câmara de refrigeração e um novo catodo primário, onde a troca de calor entre eles aumentou devido ao maior contato. A Figura 6(f) mostra o anodo secundário após um teste de limitação dos eletrodos, onde foram empregadas intensidades de corrente elétrica, nas fontes de energia primária e secundária, de 60 A e 400 A, respectivamente. A Figura 6(c) apresenta o anodo primário após todos os testes realizados com a tocha de plasma híbrida. $\mathrm{O}$ anodo primário e o catodo secundário foram os eletrodos que menos apresentaram sinais de erosão.

Também, alguns problemas de isolamento elétrico foram detectados quando a tocha de plasma híbrida operava com os arcos elétricos simultaneamente. Esta falta de isolamento elétrico gerava arcos elétricos indesejados entre flanges de cobre e eletrodos, flanges de cobre e parafusos fixadores, eletrodos e difusores, etc. Estes arcos elétricos indesejáveis acabavam danificando os componentes da tocha, tais como flanges isolantes, bocal cerâmico, parafusos fixadores, entre outros. Para evitar estes arcos elétricos foram realizadas as seguintes modificações no projeto inicial da tocha de plasma híbrida: a entrada de gás secundária foi eliminada; foi adicionada uma bucha cerâmica entre o catodo primário e o catodo secundário; buchas isolantes de nylon foram adicionadas em todos os contatos dos flanges com os parafusos; e foi adicionada uma câmara cerâmica (bocal usualmente utilizado em TIG) entre o anodo primário e o catodo secundário. Alguns componentes danificados pelas descargas (a)

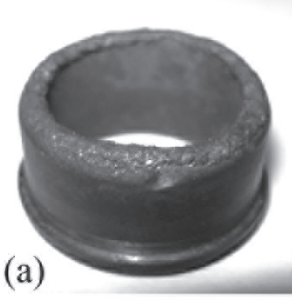

(b)

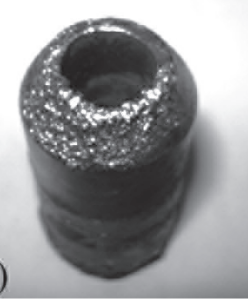

(c)
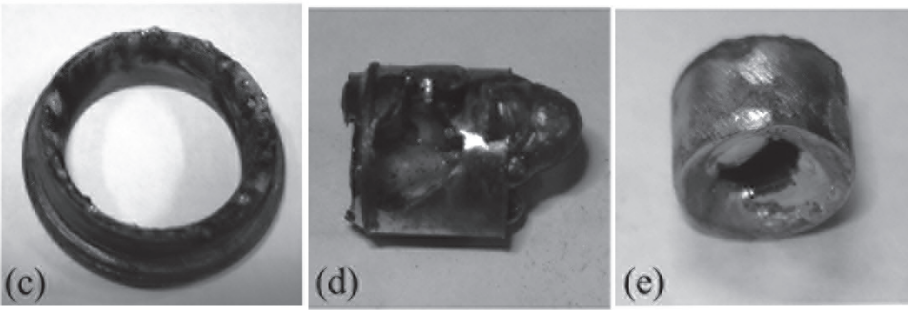

(f)
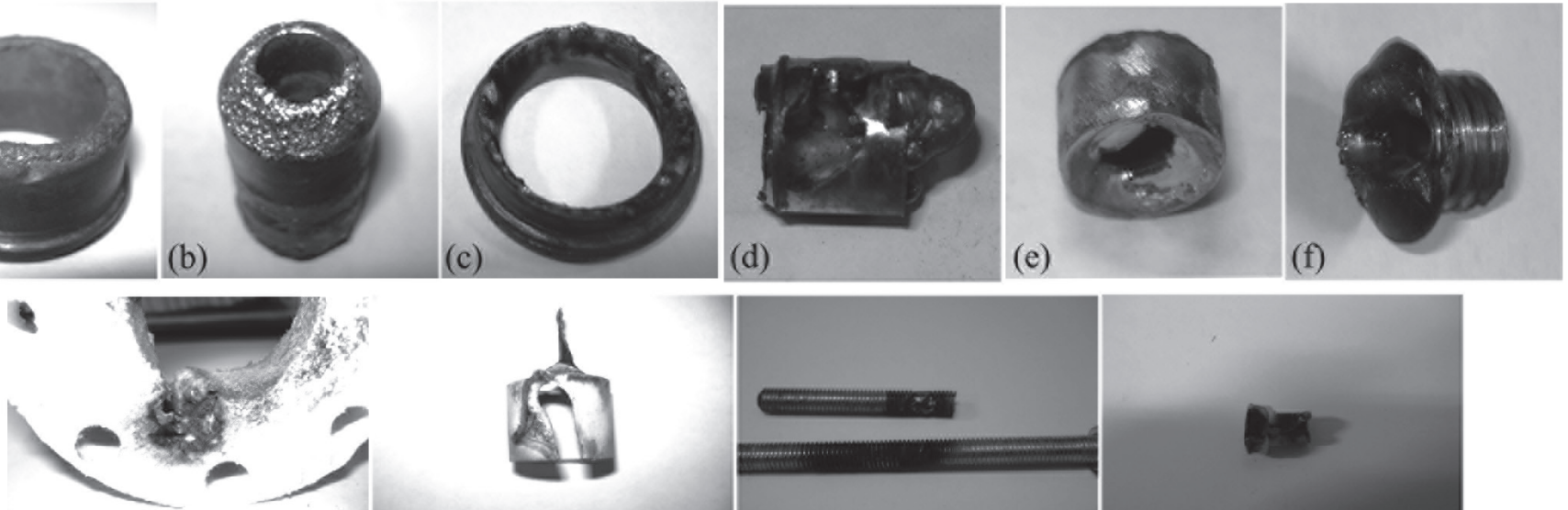

(g)

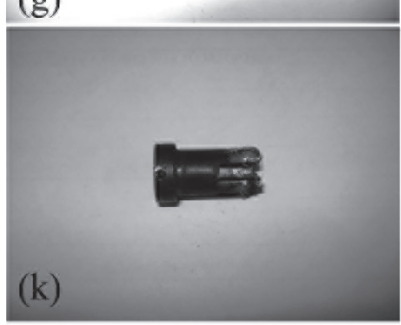

(h)
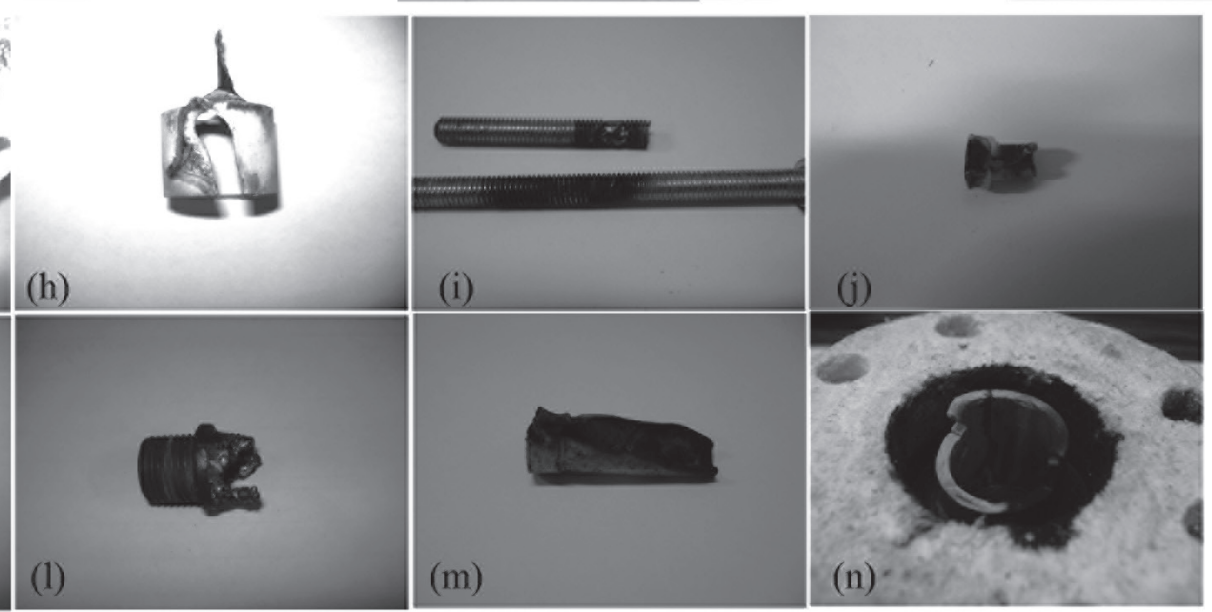

Figura 6. Eletrodos danificados pela erosão: $(a, b)$ catodos primários; $(c)$ anodo primário e $(d, e, f)$ anodos secundários. Componentes da tocha híbrida danificados por descargas elétricas: (g) flange isolante; $(\mathrm{h}, \mathrm{n})$ bocais cerâmicos; (i) parafusos; (j, $\mathrm{m}$ ) buchas isolantes; (k) pinça e (l) difusor. 

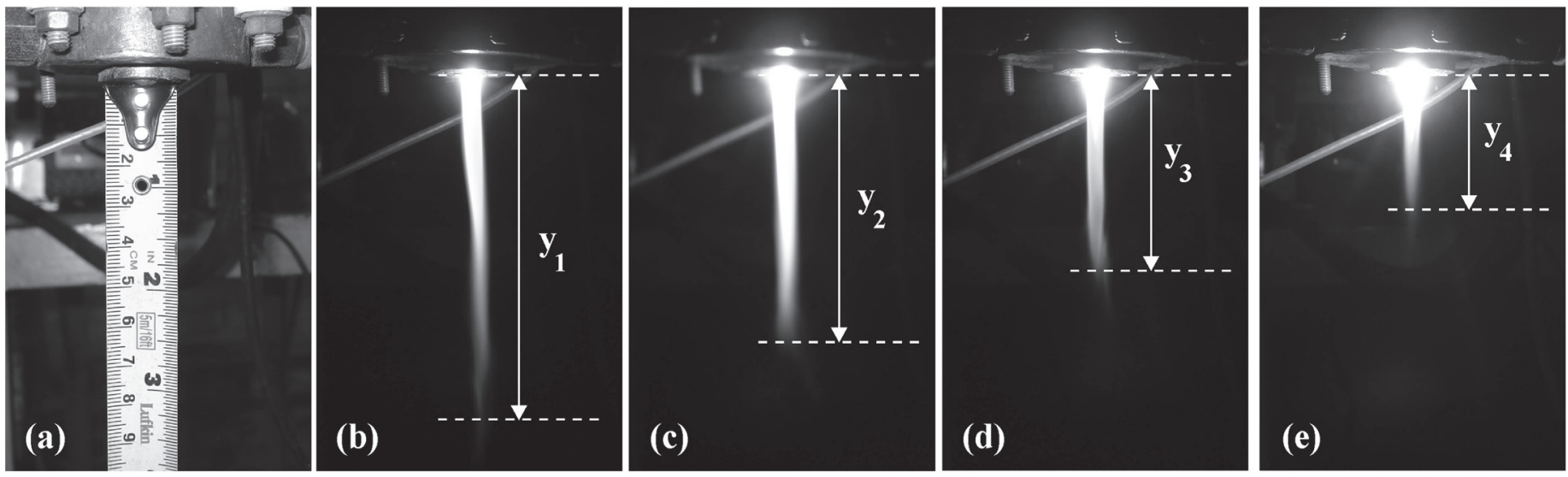

Figura 7. (a) Padrão de distância para determinar o comprimento do jato de plasma. Jatos de plasma expulso para fora da câmara, com indicação dos respectivos comprimentos, para intensidades de corrente elétrica primária de $30 \mathrm{~A}$ e secundária de $200 \mathrm{~A}$ e vazões do gás de: (b) $7 \mathrm{l} / \mathrm{min}$; (c) $14 \mathrm{l} / \mathrm{min}$; (d) $20 \mathrm{l} / \mathrm{min}$ e (e) $25 \mathrm{l} / \mathrm{min}$.

Tabela 1. Análise de variância (ANOVA) para o comprimento do jato de plasma.

\begin{tabular}{|c|c|c|c|c|c|}
\hline Fonte & Soma dos Quadrados & Grau de Liberdade & $\begin{array}{c}\text { Médias dos } \\
\text { Quadrados }\end{array}$ & Valor de F & P - Valor \\
\hline$I_{P}$ & 639,9 & 3 & 213,3 & 4,3 & 0,006 \\
\hline$I_{S}$ & 18449,3 & 3 & 6149,8 & 122,5 & 0,000 \\
\hline$V_{g}$ & 16315,2 & 3 & 5438,4 & 108,3 & 0,000 \\
\hline Erro & 9139,3 & 182 & 50,2 & ---- & ---- \\
\hline Total & 44543,7 & 191 & ---- & ---- & --- \\
\hline
\end{tabular}

elétricas podem ser visualizados nas Figuras 6(g-n). Portanto, várias modificações do projeto inicial foram necessárias para se obter funcionamento estável da tocha de plasma híbrida.

\subsection{Comprimento do Jato de Plasma}

O padrão de distância para determinar o comprimento do jato de plasma expulso para fora da câmara é apresentado na Figura 7(a). Por sua vez, as Figuras 7(b-e) mostram uma sequência de fotos, nas quais os comprimentos $\mathrm{y}_{1} \ldots \mathrm{y}_{4}$ dos jatos de plasma são representados. Estes jatos de plasma foram produzidos com os seguintes parâmetros: intensidade da corrente elétrica primária de $30 \mathrm{~A}$; intensidade da corrente elétrica secundária de 200 A e vazões do gás de 7 1/min (Figura 7(b)), 14 1/min (Figura 7(c)), 20 1/min (Figura 7(d)) e 25 1/min (Figura 7(e)). Através das imagens, observa-se que o comprimento do jato de plasma diminui com o aumento da vazão do gás e isto foi verificado em todos os experimentos.

A Tabela 1 apresenta os resultados da análise de variância, com nível de confiabilidade de $95 \%$, para comprimento do jato de plasma expulso para fora da câmara. Através desta análise, observa-se que os valores de probabilidade P-valor são menores que 0,05 e, consequentemente, com $95 \%$ de confiabilidade podese dizer que todas as variáveis tiveram influência significativa sobre comprimento do jato de plasma. Também, as variáveis que tiveram maior influência sobre o comprimento do jato de plasma podem ser determinadas pelos valores de $\mathrm{F}$, isto é, quanto maior o valor de F maior será a influência. Então, as variáveis mais influentes sobre o comprimento do jato de plasma foram a intensidade da corrente elétrica secundária e a vazão do gás.

A Figura 8(a-d) apresenta os gráficos do comprimento do jato de plasma em função da intensidade de corrente elétrica primária para diferentes vazões de gás. As intensidades de corrente elétrica entre os eletrodos secundários foram, respectivamente, de $100 \mathrm{~A}, 150 \mathrm{~A}, 200 \mathrm{~A}$ e $250 \mathrm{~A}$. Estes gráficos mostram que o comprimento do jato de plasma sofreu pequenas variações em função da intensidade de corrente elétrica primária e diminuiu com o aumento da vazão do gás.

A Figura 9(a-d) apresenta os gráficos do comprimento do jato de plasma em função da intensidade de corrente elétrica secundária para diferentes vazões de gás. As intensidades de corrente elétrica entre os eletrodos primários foram, respectivamente, de $30 \mathrm{~A}, 40 \mathrm{~A}, 50 \mathrm{~A}$ e $60 \mathrm{~A}$. Estes gráficos mostram que, em média, o comprimento do jato de plasma aumentou com o aumento da intensidade de corrente elétrica secundária e diminuiu com o aumento da vazão do gás.

Os resultados obtidos na caracterização, em relação ao comprimento do jato de plasma, mostraram que as intensidades da corrente elétrica (primária e secundária) e a vazão do gás provocam alterações significativas no comprimento do jato de plasma. As características observadas para os jatos de plasma gerados pela tocha de plasma híbrida são semelhantes aos resultados obtidos por Pan et al. [17, 18], ou seja, o comprimento do jato de plasma aumenta com a elevação da intensidade de 


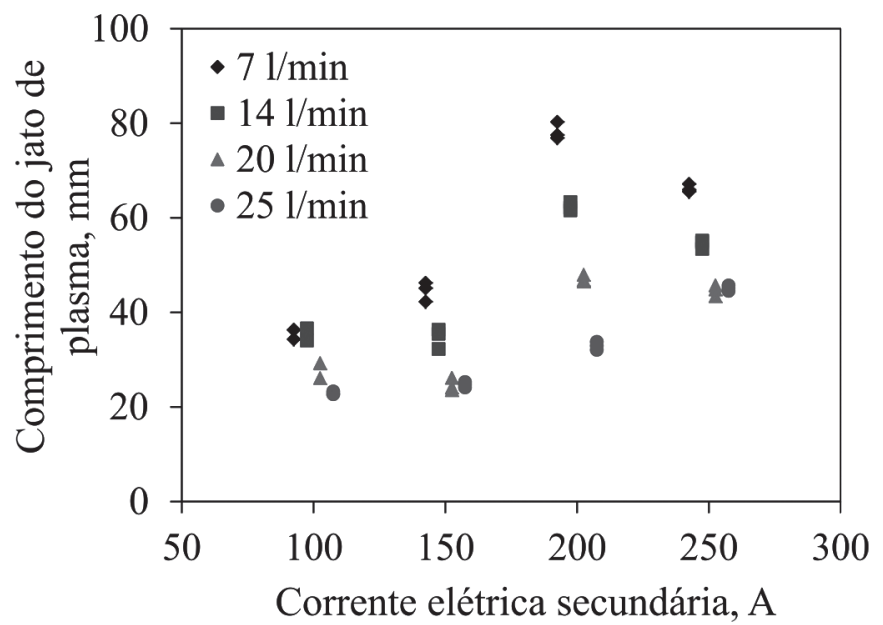

(a)

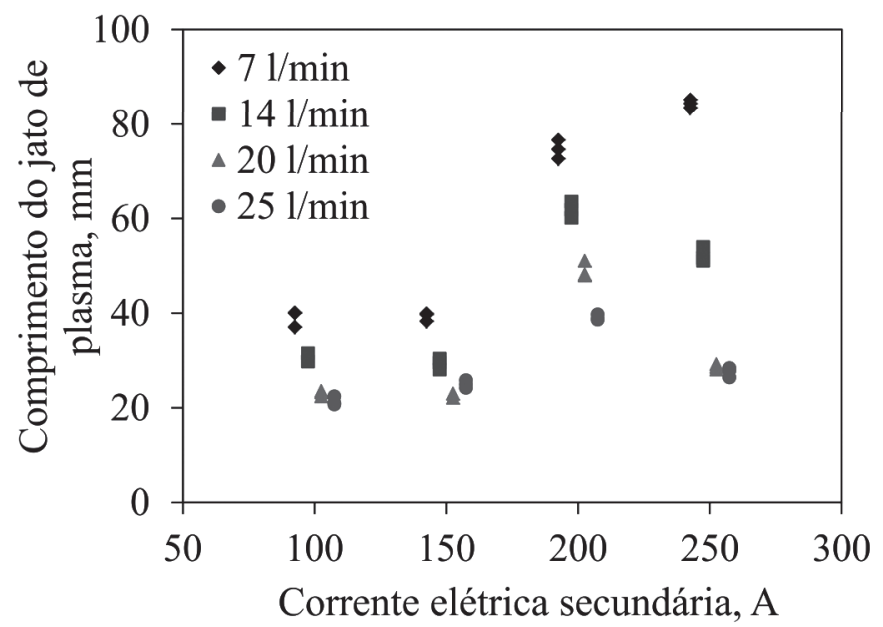

(c)

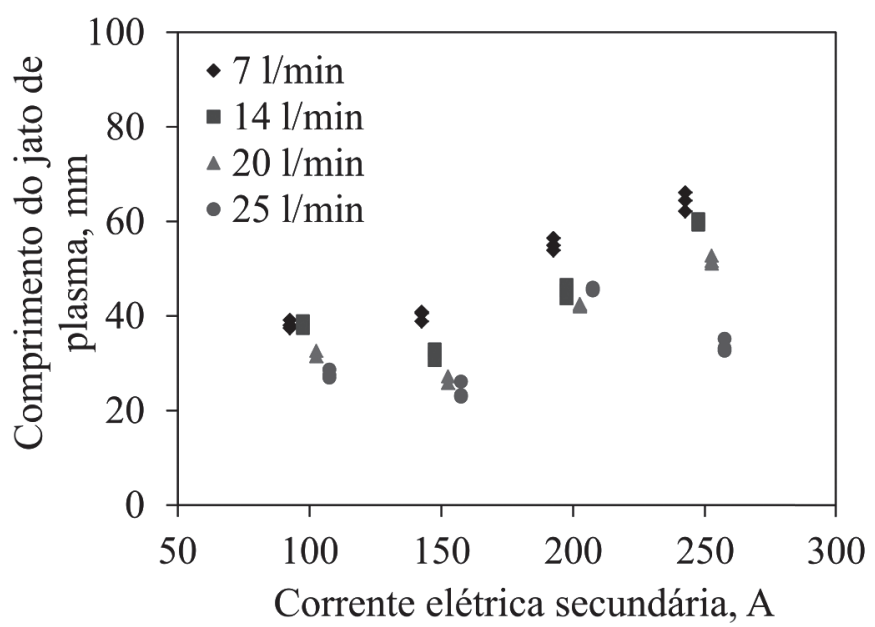

(b)

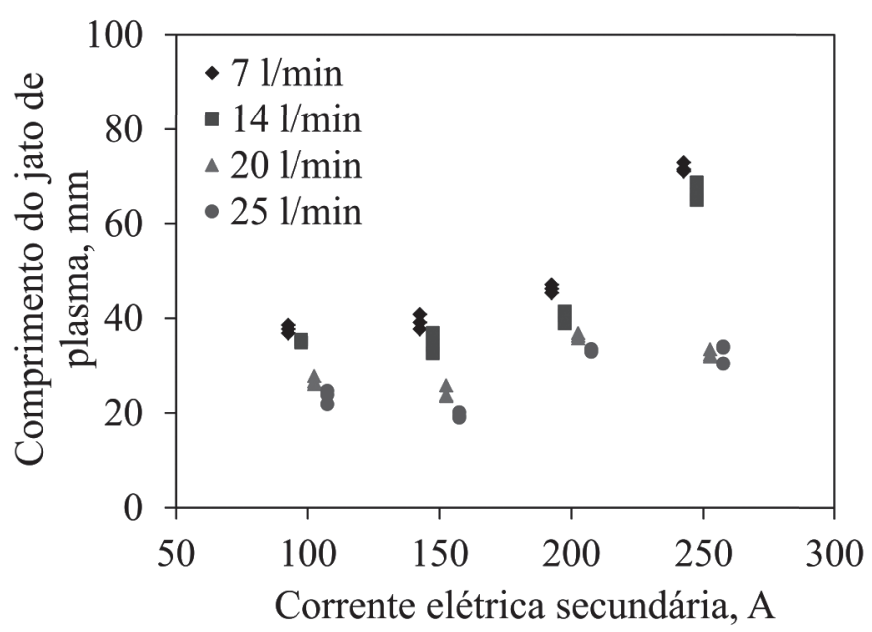

(d)

Figura 8. Comprimento do jato de plasma em função da intensidade de corrente elétrica primária para diferentes vazões de gás e intensidade de corrente elétrica secundária de (a) $100 \mathrm{~A}$, (b) $150 \mathrm{~A}$, (c) $200 \mathrm{~A} \mathrm{e} \mathrm{(d)} 250 \mathrm{~A}$.

corrente elétrica secundária e diminui com o aumento da vazão do gás (intervalo de transição do fluxo de plasma laminar para turbulento). Porém, uma exceção é relativa aos resultados obtidos para a intensidade da corrente elétrica primária, onde o comprimento do jato de plasma tem uma pequena tendência decrescente com o seu aumento. Com base em outros estudos $[17,18]$, é possível afirmar que a diminuição do comprimento do jato de plasma com o aumento da vazão do gás, mantendo-se constantes as intensidades de corrente elétrica entre os eletrodos (primários e secundários), deve-se ao fato das forças viscosas de cisalhamento —as quais dificultam o movimento relativo entre as camadas adjacentes do jato de plasma- não estarem amortecendo as tendências de instabilidades e turbulência, isto é, ocorre uma transição de fluxo laminar para um fluxo turbulento devido a possível existência de um número de Reynolds crítico, o qual é diretamente proporcional à vazão do gás e inversamente proporcional à viscosidade. Também, é possível dizer que a viscosidade do gás argônio varia com o aumento da temperatura do mesmo, o qual é oriundo do aumento das intensidades de corrente elétrica entre os eletrodos (primários e secundários).
Então, a variação do número Reynolds e, consequentemente, a variação do comprimento do jato de plasma pode ser realizada através das variações dos parâmetros de operação da tocha (intensidades de corrente elétrica entre os eletrodos e vazão do gás).

\subsection{Soldagem e Corte com a Tocha de Plasma Híbrida}

Os resultados obtidos para a aplicação do dispositivo em processos de fabricação foram satisfatórios, isto é, foi possível realizar soldagem e corte com a tocha de plasma híbrida. Porém, estudos para otimização destes procedimentos com esta tocha devem ser ainda realizados.

A Figura 10 mostra (vista superior) chapas submetidas à soldagem com a tocha de plasma híbrida, onde os números indicados nas fotografias correspondem aos números dos testes de soldagem apresentados na Tabela 2, a qual também apresenta os parâmetros de soldagem utilizados. Em todos os testes, a distância entre o bocal e a peça foi de $5 \mathrm{~mm}$ e a intensidade da corrente elétrica primária de $60 \mathrm{~A}$. O dispositivo 


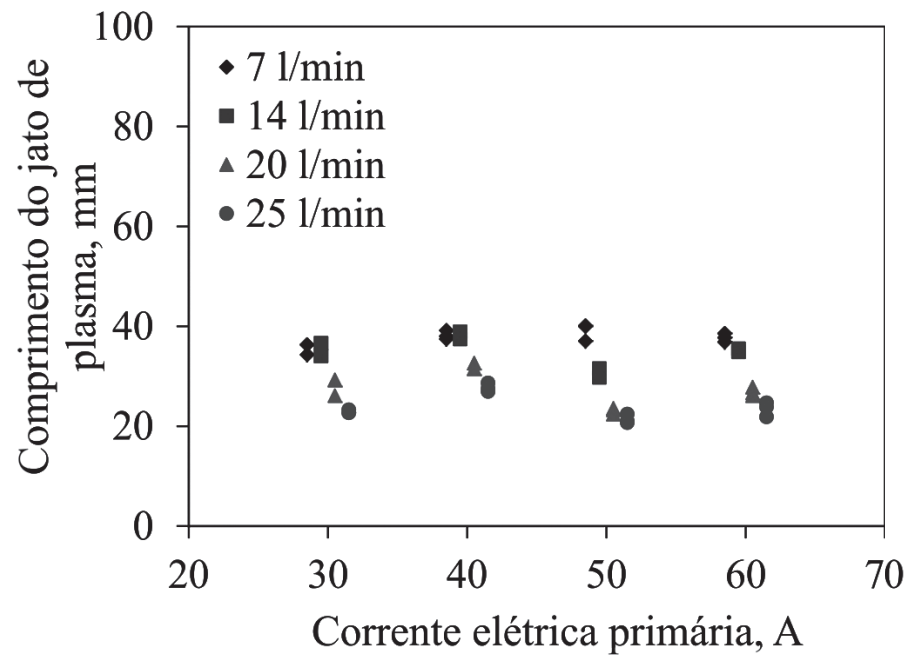

(a)

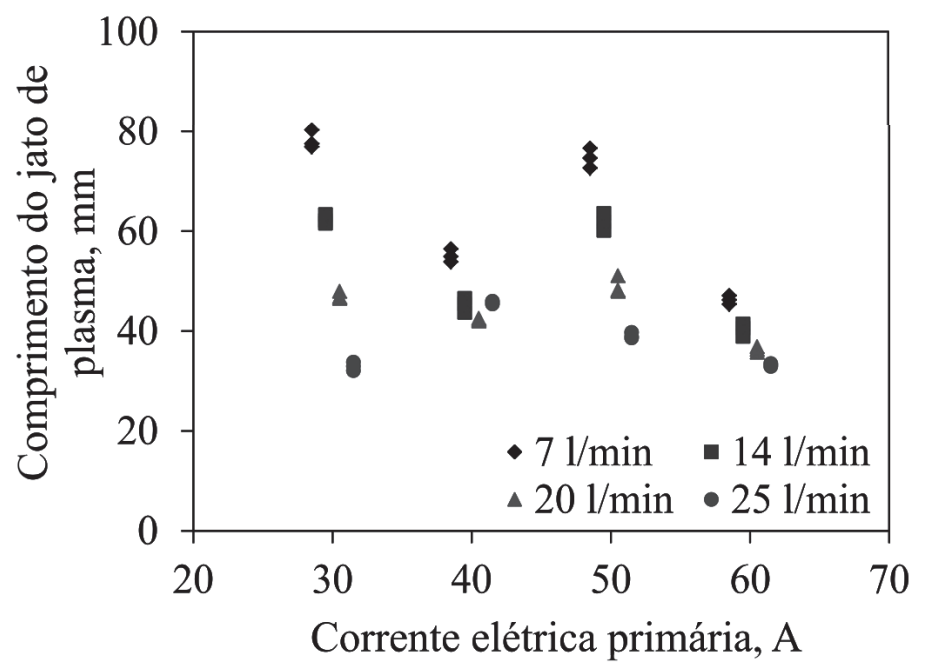

(c)

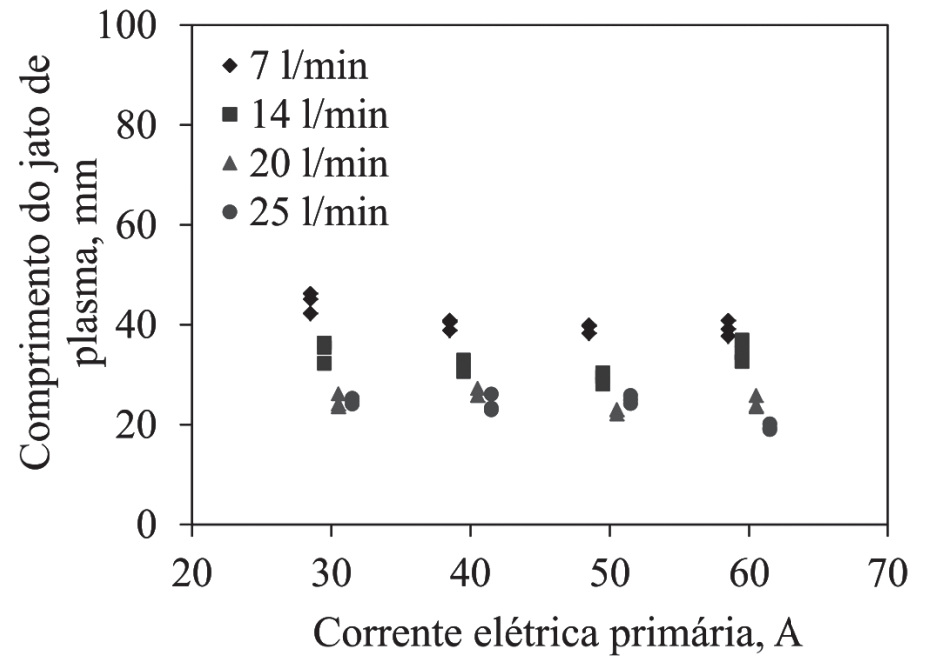

(b)

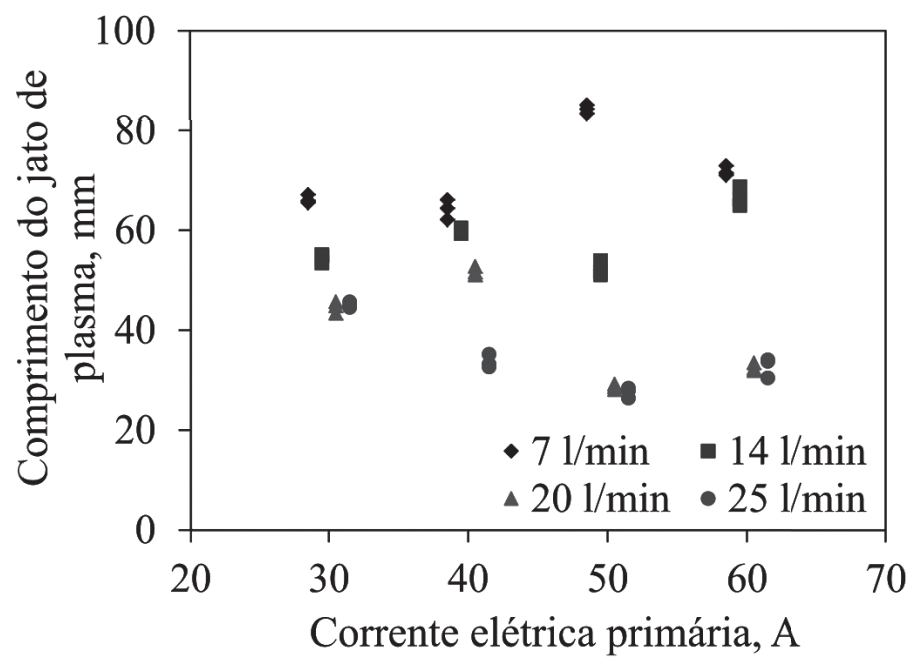

(d)

Figura 9. Comprimento do jato de plasma em função da intensidade de corrente elétrica secundária para diferentes vazões de gás e intensidade de corrente elétrica primária de (a) $30 \mathrm{~A}$, (b) $40 \mathrm{~A}$, (c) $50 \mathrm{~A} \mathrm{e} \mathrm{(d)} 60 \mathrm{~A}$.

Tabela 2. Experimentos realizados com respectivos parâmetros de soldagem.

\begin{tabular}{|c|c|c|c|c|c|}
\hline \multirow{2}{*}{ Parâmetros } & \multicolumn{5}{|c|}{ Testes Experimentais } \\
\hline & 1 & 2 & 3 & 4 & 5 \\
\hline$I_{S}-\mathrm{A}$ & 200 & 200 & 200 & 200 & 250 \\
\hline Vazão do Gás - 1/min & 7 & 14 & 7 & 7 & 25 \\
\hline $\begin{array}{c}\text { Velocidade de } \\
\text { Soldagem }-\mathrm{mm} / \mathrm{s}\end{array}$ & 1,7 & 1,7 & 3,4 & 1,7 & 1,7 \\
\hline Tipo de Aço & AISI 304 & AISI 304 & AISI 304 & AISI 304 & SAE 1020 \\
\hline $\begin{array}{l}\text { Dimensões das } \\
\text { Chapas - mm }\end{array}$ & $100 \times 50 \times 1,5$ & $100 \times 50 \times 1,5$ & $100 \times 50 \times 1,5$ & $100 \times 50 \times 0,7$ & $200 \times 50 \times 6,35$ \\
\hline
\end{tabular}




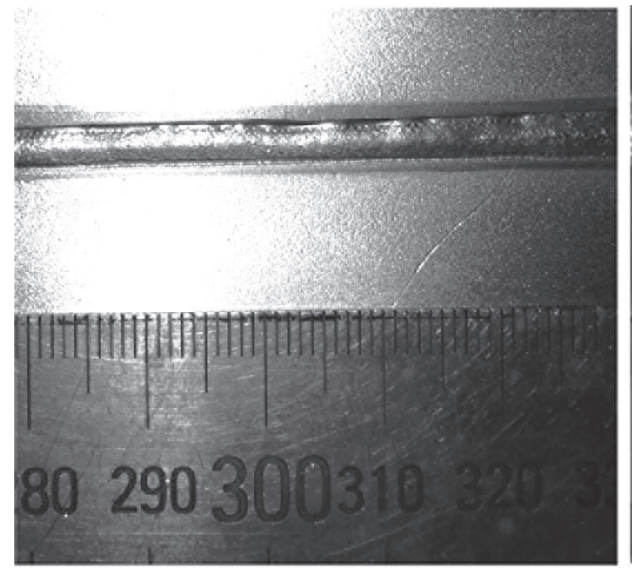

(1)

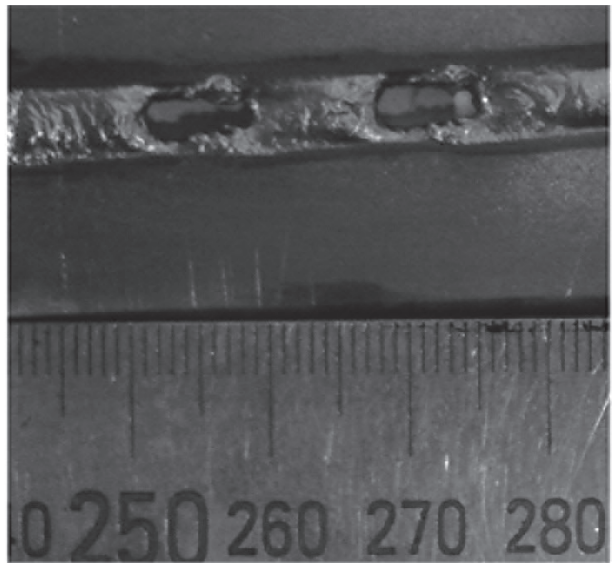

(2)

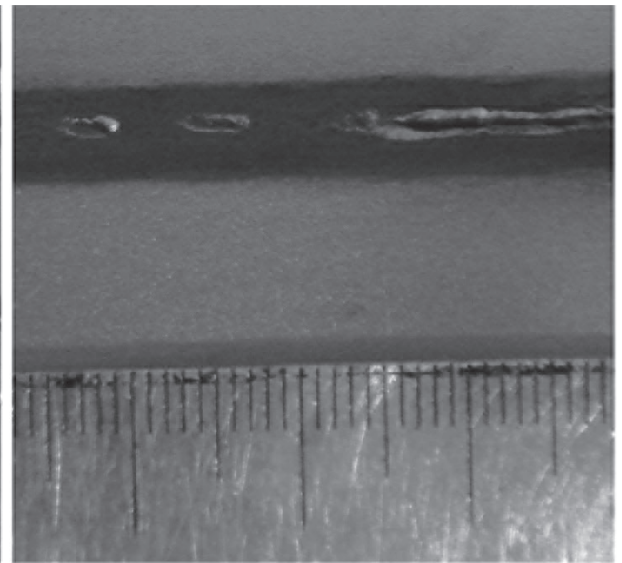

(3)

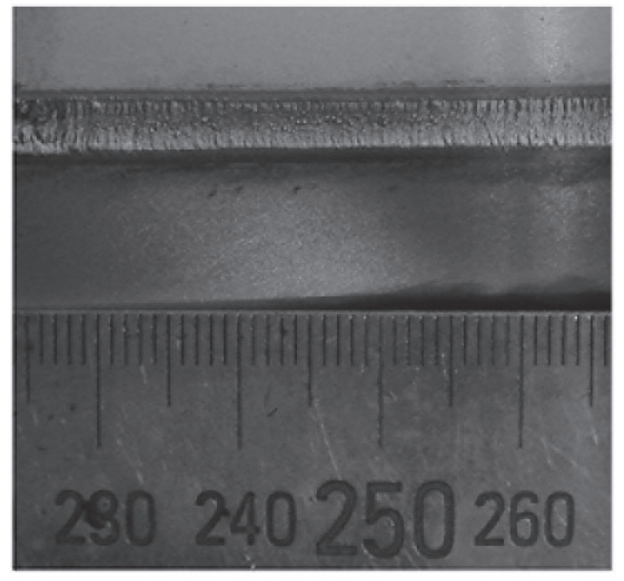

(4)

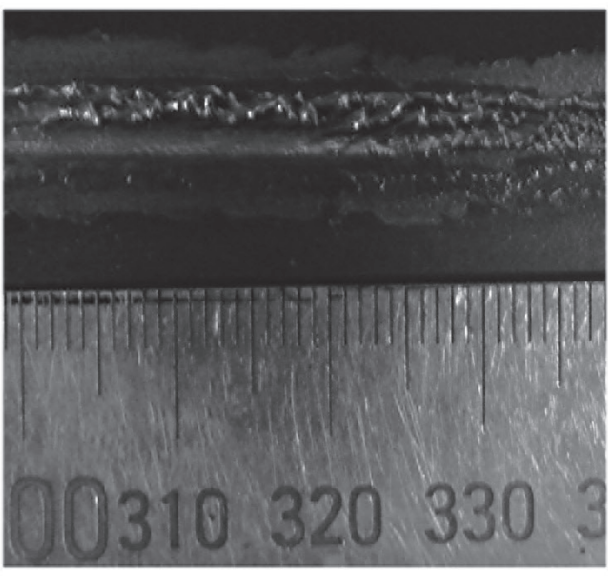

(5)

Figura 10. Vista superior das chapas de aço soldadas.

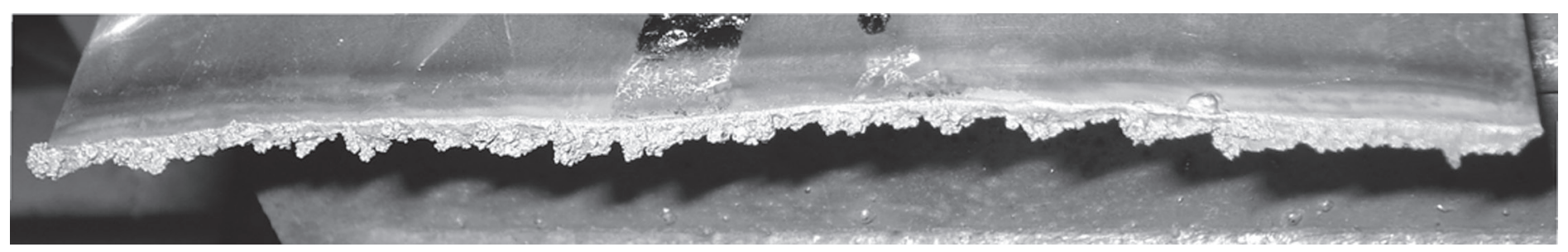

Figura 11. Vista lateral do corte de uma chapa de aço realizado com a tocha de plasma híbrida.

mostrou-se capaz de realizar soldagem de chapas finas $(0,7$ e $1,5 \mathrm{~mm}$ ) com vazões de gás iguais ou menores a $71 / \mathrm{min}$, pois com vazões maiores o jato de plasma tornava-se turbulento e formava cordões de solda irregulares (testes 2 e 5 ). $\mathrm{O}$ teste 3 apresenta algumas falhas de fusão devido a elevada velocidade de soldagem empregada para esta tocha.

Os cortes realizados com o dispositivo apresentaram irregularidades devido ao jato de plasma não ter força suficiente para expulsar o metal fundido, não sendo possível cortar chapas com espessuras acima de $3 \mathrm{~mm}$. A Figura 11 mostra secção transversal de uma chapa de aço AISI 304 com espessura de 1,5 $\mathrm{mm}$ cortada com a tocha híbrida geradora de plasma. O corte foi realizado com os seguintes parâmetros: intensidade de corrente elétrica primária de $60 \mathrm{~A}$ e secundária de $250 \mathrm{~A}$; vazão do gás de
$25 \mathrm{l} / \mathrm{min}$; velocidade de corte de $8.4 \mathrm{~mm} / \mathrm{s}$ e distância do bocal à peça de $5 \mathrm{~mm}$.

Para aumentar a eficiência e a qualidade do corte, possivelmente a elevação da vazão do gás provocaria completa expulsão do metal fundido; combinando este fator com maiores intensidades da corrente elétrica, resultaria em altas velocidades de corte e a possibilidade do corte de chapas com maiores espessuras.

\section{Conclusões}

De acordo com os resultados obtidos nos experimentos e as análises realizadas, as seguintes conclusões podem ser apresentadas: 
- Foi projetada, construída, testada e aperfeiçoada uma tocha de plasma híbrida, a qual caracteriza-se pela formação simultânea de dois arcos plasmas em apenas um dispositivo, resultante da combinação sinérgica de dois processos geradores de plasma, ou seja, Plasma Não-Transferido e Propulsor Magnetoplasmadinâmico.

- As três variáveis (intensidades de corrente elétrica, primária e secundária, e vazão do gás) investigadas apresentaram influência significativa, com intervalo de confiança de $95 \%$, sobre o comprimento do jato de plasma, onde o mesmo aumenta com o aumento da intensidade de corrente elétrica secundária e sofre uma leve diminuição com o aumento da intensidade de corrente elétrica primária. Também, aumentando a vazão do gás, de $7 \mathrm{l} / \mathrm{min}$ para $25 \mathrm{l} / \mathrm{min}$, o comprimento do jato de plasma diminui.

- O dispositivo mostrou-se capaz de realizar soldagem de chapas finas com vazão de gás igual ou menor do que 7 1/min, pois com vazão acima de $7 \mathrm{1} / \mathrm{min}$ o jato de plasma tornava-se turbulento e formava cordões de solda irregulares.

- Os cortes realizados com o dispositivo apresentaram irregularidades devido ao jato de plasma não ter força suficiente para expulsar o metal fundido. Não foi possível cortar chapas de aço com espessuras acima de $3 \mathrm{~mm}$.

\section{Agradecimentos}

Um dos autores (R.T.L.) agradece ao CNPq pela bolsa de estudo concedida durante seu Doutorado no PPGE3M, realizado no LS\&TC.

\section{Referências Bibliográficas}

[1] ROTH, J. R. Industrial Plasma Engineering. Volume 1: Principles. Institute of Physics Publishing, v. 1, Philadelphia, 1995.

[2] ZHUKOV, M. F. and ZASYPKIN, I. M. Thermal Plasma Torches -Design, Characteristics, Applications. Cambridge International Science Publishing, 2007.

[3] MESSLER, R. W. J. What's Next for Hybrid Welding. Welding Journal, 83(3): 30-34, Mar 2004.

[4] ESSERS, W.G.; WILLEMS, G.A.M.; BUELENS, J.J.C. and VAN GOMPEL, M.R.M. Plasma-MIG Welding -A New Torch and Arc Starting Method. Metal Construction, (1): 36-42, Jan 1981

[5] TU, X.; CHÉRON, B.G.; YAN, J. H. and CEN, K.F. Electrical and Spectroscopic Diagnostic of an Atmospheric Double Arc Argon Plasma Jet. Plasma Sources Science and Technology, 16: 803-812, 2007.

[6] TU, X.; YU, L.; YAN, J. H.; CEN, K. and CHÉRON, B. Heat Flux Characteristics in an Atmospheric Double Arc Argon Plasma Jet. Applied Physics Letters, 93(15), Oct 2008.

[7] BROWNING, J. A. Hybrid Non-Transferred-Arc PlasmaTorch System and Method of Operating Same. Patent US 4,626,648, December 2, 1986.

[8] Welding Handbook. Vol. II - Welding Process. $8^{\text {th }}$ ed., American Welding Society, Miami, Fl, 1991.
[9] MACHADO, I. G. Soldagem \& Técnicas Conexas: Processos. Editado pelo Autor, Distribuído pela Associação Brasileira de Soldagem, Porto Alegre, 1996. Edição esgotada: disponível gratuitamente no formato ".pdf” em www.ct.ufrgs. br/lstc/.

[10] KOU, S. Welding Metallurgy. John Wiley \& Sons, Inc, $2^{\text {nd }}$ ed., 2002.

[11] MACHADO, I. G. and LERMEN, R. T. Development and Implementation of a Magnetoplasmadynamic Thruster for Welding and Cutting. Journal of Materials Processing Technology, 200: 398-404, 2008.

[12] LERMEN, R. T. Desenvolvimento de uma tocha de plasma híbrida para o processamento de materiais. Tese de Doutorado. Universidade Federal do Rio Grande do Sul (UFRGS), 2011.

[13] JAHN, R. G. Physics of Electric Propulsion. McGraw-Hill Company, New York, 1968.

[14] TILLACK, M. S. and MORLEY, N. B. MagnetoHydrodynamic. McGraw Hill Standard Handbook for Electrical Engineers, 14 ${ }^{\text {th }}$ ed., 1998.

[15] BATCHELOR, G. K. An Introduction to Fluid Dynamics. Cambridge University Press, 2000.

[16] SOLONENKO, O. P. Thermal Plasma Torches and Technologies. Cambridge International Science Publishing, 2003.

[17] PAN, W.; ZHANG, W.; MA, W. and WU, C. Characteristics of Argon Laminar DC Plasma Jet at Atmospheric Pressure. Plasma Chemistry and Plasma Processing, 22(2): 271-283, Jun 2002.

[18] PAN, W. X.; MENG, X.; LI, G.; FEI, Q. X. and WU, C. K. Feasibility of Laminar Plasma-Jet Hardening of Cast Iron Surface. Surface \& Coatings Technology, 197(2-3): 345-350, Jul 2005. 